Research in Astron. Astrophys. Vol.0 (200x) No.0, 000-000

http://www.raa-journal.org http://www.iop.org/journals/raa

$\boldsymbol{R}$ esearch in

A stronomy and

Astrophysics

\title{
Determination of the Magnetic Fields of Magellanic X-Ray Pulsars
}

\author{
Dimitris M. Christodoulou ${ }^{1,2}$, Silas G. T. Laycock ${ }^{1,3}$, Jun Yang ${ }^{1,3}$, and Samuel Fingerman ${ }^{1,3}$ \\ ${ }^{1}$ Lowell Center for Space Science and Technology, University of Massachusetts Lowell, Lowell, MA, \\ 01854, USA. \\ 2 Department of Mathematical Sciences, University of Massachusetts Lowell, Lowell, MA, 01854, \\ USA. E-mail: dimitris_christodoulou@uml.edu \\ 3 Department of Physics \& Applied Physics, University of Massachusetts Lowell, Lowell, MA, 01854, \\ USA. E-mail: silas_laycock@uml.edu, jun_yang@uml.edu, fingerman.samuel@gmail.com
}

\begin{abstract}
The 80 high-mass X-ray binary (HMXB) pulsars that are known to reside in the Magellanic Clouds (MCs) have been observed by the XMM-Newton and Chandra X-ray telescopes on a regular basis for 15 years, and the XMM-Newton and Chandra archives contain nearly complete information about the duty cycles of the sources with spin periods $P_{S}<100 \mathrm{~s}$. We have rerprocessed the archival data from both observatories and we combined the output products with all the published observations of $31 \mathrm{MC}$ pulsars with $P_{S}<100 \mathrm{~s}$ in an attempt to investigate the faintest X-ray emission states of these objects that occur when accretion to the polar caps proceeds at the smallest possible rates. These states determine the so-called propeller lines of the accreting pulsars and yield information about the magnitudes of their surface magnetic fields. We have found that the faintest states of the pulsars segregate into five discrete groups which obey to a high degree of accuracy the theoretical relation between spin period and X-ray luminosity. So the entire population of these pulsars can be described by just five propeller lines and the five corresponding magnetic moments $\left(0.29,0.53,1.2,2.9\right.$, and 7.3 , in units of $\left.10^{30} \mathrm{G} \mathrm{cm}^{3}\right)$.
\end{abstract}

Key words: Magellanic clouds—accretion, accretion disks—stars: magnetic field—stars: neutron-X-rays: binaries

\section{INTRODUCTION AND MOTIVATION}

The XMM-Newton and Chandra X-ray observatories have monitored the Magellanic Clouds (MCs) on a regular basis in the years 2000-2014 and their archives contain a wealth of information about the 80 $\mathrm{X}$-ray sources that were identified to be members of high-mass X-ray binaries (HMXBs). We have reanalyzed all Magellanic data from both archives and we created a new pipeline of products that delineate the physical properties of HMXBs and their accreting pulsars. The details of our analysis, the resulting library, and the public release of the raw and processed data are described in the works of Yang et al. (2017) and Christodoulou et al. (2016). In this investigation, we extend the work of Christodoulou et al. (2016) who focused on the faintest X-ray observations in order to map out the lowest states of pulsed $\mathrm{X}$-ray emission that occur when accretion to the polar caps proceeds at the smallest possible rates. Of the entire population of X-ray pulsars, only eight of them (lowest row in Table 1) were observed in such states and their lowest-luminosity observations appeared to define the absolutely lowest propeller line in the spin period-luminosity $\left(P_{S}-L_{X}\right)$ diagram of the MC pulsars. The best-fit line through these eight points turned out to be in excellent agreement with the theoretical propeller line, as this was derived by Stella et al. (1986), and it yielded a canonical magnitude for the surface magnetic field of these pulsars of $B=3 \times 10^{11} \mathrm{G}$ (or a magnetic moment of $\mu=3 \times 10^{29} \mathrm{G} \mathrm{cm}^{3}$ ). 


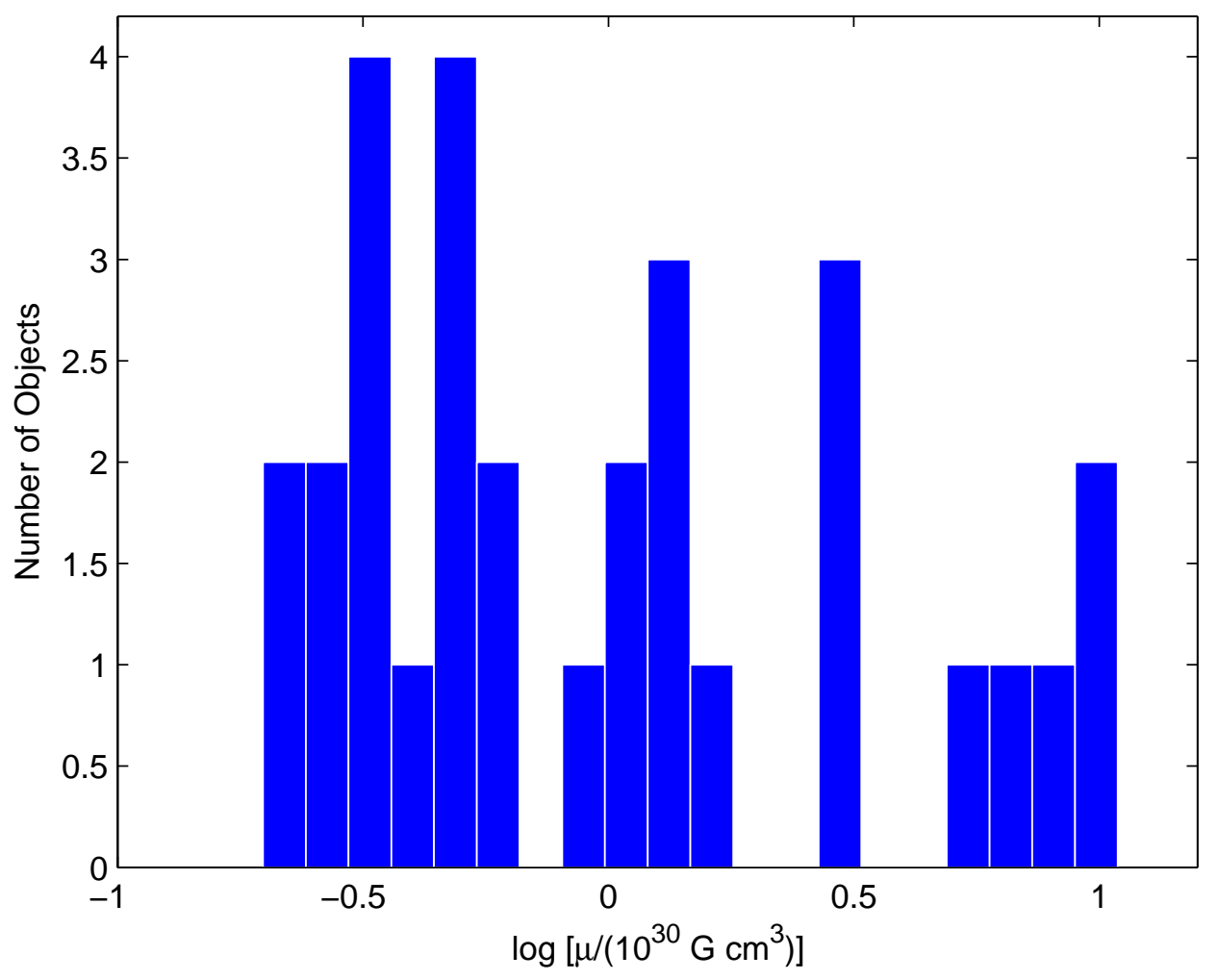

Fig. 1 Histogram of $\log \mu$ values obtained from equation (1) for the Magellanic pulsars with spin periods $P_{S}<100 \mathrm{~s}$. The data were presented in Christodoulou et al. (2016). The raw data was distributed in 20 equally spaced bins between the values -0.704 and 1.04. Five peaks are readily visible and the pulsars that cluster around each peak are listed in Table 1 .

Table 1 Discrete Propeller Lines in the $P_{S}-L_{X}$ Diagram

\begin{tabular}{ccc}
\hline \hline $\begin{array}{c}\text { Propeller } \\
\text { Line }\end{array}$ & $\begin{array}{c}\text { Number } \\
\text { of MC Pulsars }\end{array}$ & $\begin{array}{c}\text { Contributing } \\
\text { MC Pulsars }\end{array}$ \\
\hline Highest & 5 & SXP: 4.78, 6.85, 74.7 and LXP: 28.8, 61.6 \\
Fourth & 3 & SXP: $5.05,7.78,59.0$ \\
Third & 7 & SXP: $2.37,8.02,25.5,31.0,46.6,82.4$ and LXP: 4.40 \\
Second & 7 & SXP: $0.72,7.92,9.13,11.6,11.87,15.3$ and CXOU J010043.1-721134 \\
Lowest & 8 & SXP: $3.34,6.88,8.88,18.3,22.1$ and LXP: $0.07,4.10,8.04$ \\
\hline
\end{tabular}

The rest of the X-ray sources did not appear to reach down to the lowest propeller line despite the fact that the duty cycles of short-period pulsars (with $P_{S}<100 \mathrm{~s}$ ) were covered very well by both observatories over a period of 15 years. This implies that these pulsars have stronger magnetic fields and their faintest states are lying significantly higher than the lowest propeller line determined by Christodoulou et al. (2016). In this work, we adopt again the assumption that the minimum X-ray luminosities of these sources will not be randomly distributed in the $P_{S}-L_{X}$ diagram; instead, they will cluster in groups, each characterized by a typical higher value of their magnetic fields, owing to the similarities of these accreting pulsars in their structures and in their evolutionary paths. We searched for 


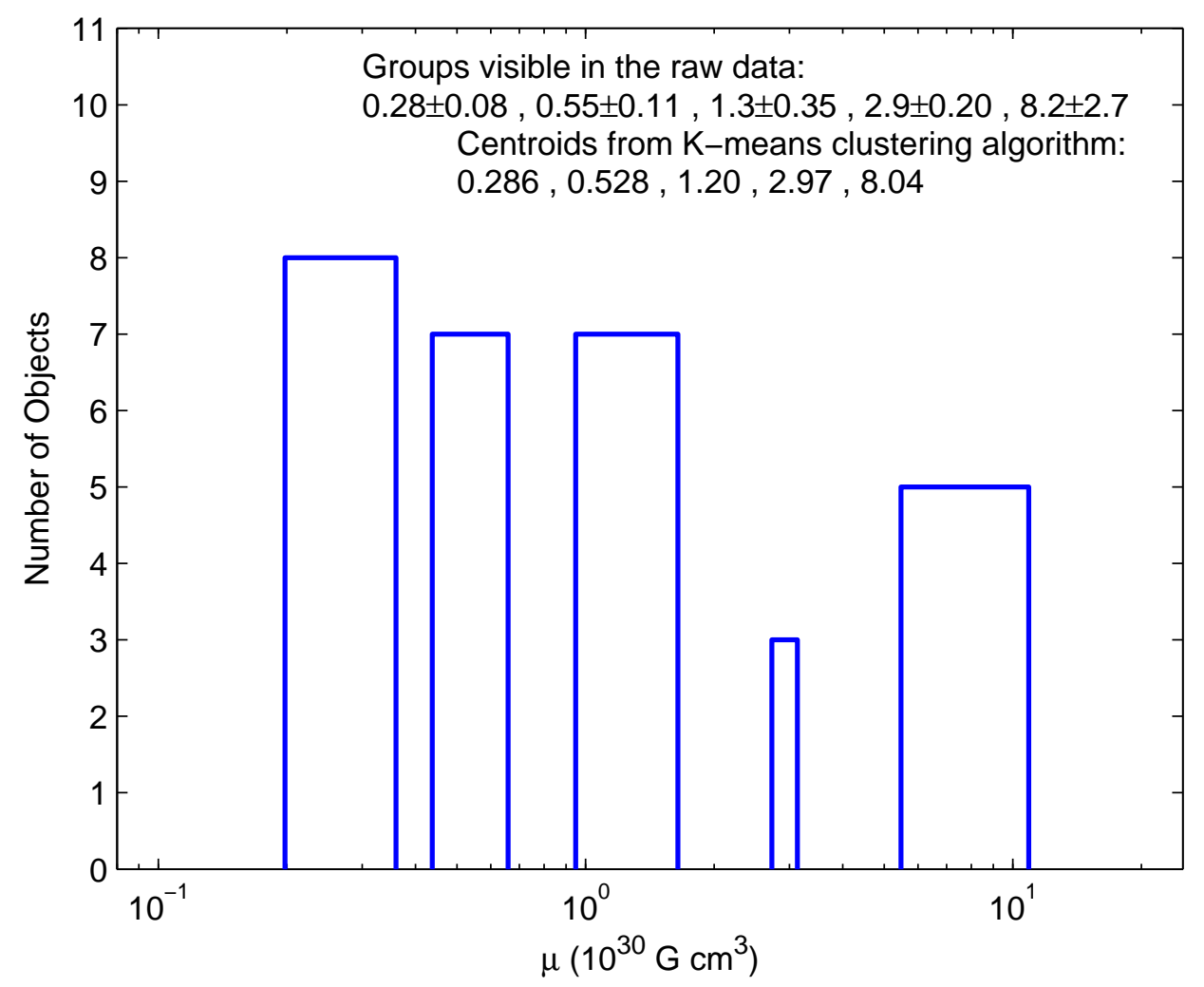

Fig. 2 Histogram of the magnetic moments $\mu$ obtained from equation (1) for the sources listed in Table 1 The data were presented in Christodoulou et al. (2016). The gaps between bars are very large, comparable to or larger than the half-widths of the bars. A K-means clustering algorithm minimizing Euclidean squared distances also confirms the observed clusters and shows that there is no overlap between clusters (see Figure 3). It is clear that the magnetic fields of the Magellanic pulsars are segregated into five distinct groups, as specified in the legend and in Table 1 .

such progressively higher values of the magnetic fields in the lowest-power observations of the X-ray sources listed in Tables 1-3 of Christodoulou et al. (2016). A histogram of $\log \mu$ values distributed in 20 equally spaced bins is shown in Figure 1. Five peaks are visible in the raw data, indicating that it is worth pursuing a formal clustering analysis. The pulsars that cluster around each peak are shown in Table1.

\subsection{Clustering Analysis}

The five groups of magnetic moments and their boundaries are represented in Figure 2 using bins centered around the peaks in Fig. 11. One can specify these bins in the raw data because of the uncharacteristically large gaps between groups. Furthermore, an investigation of clustering in the data using the K-means algorithm (Seber 1984; Spath 1985) and minimizing Euclidean squared distances confirms the dense clustering of these five groups. The centroids of the clusters are shown in the legend of Figure 2 , and they are in excellent agreement with the groups found by visual inspection of the data. 


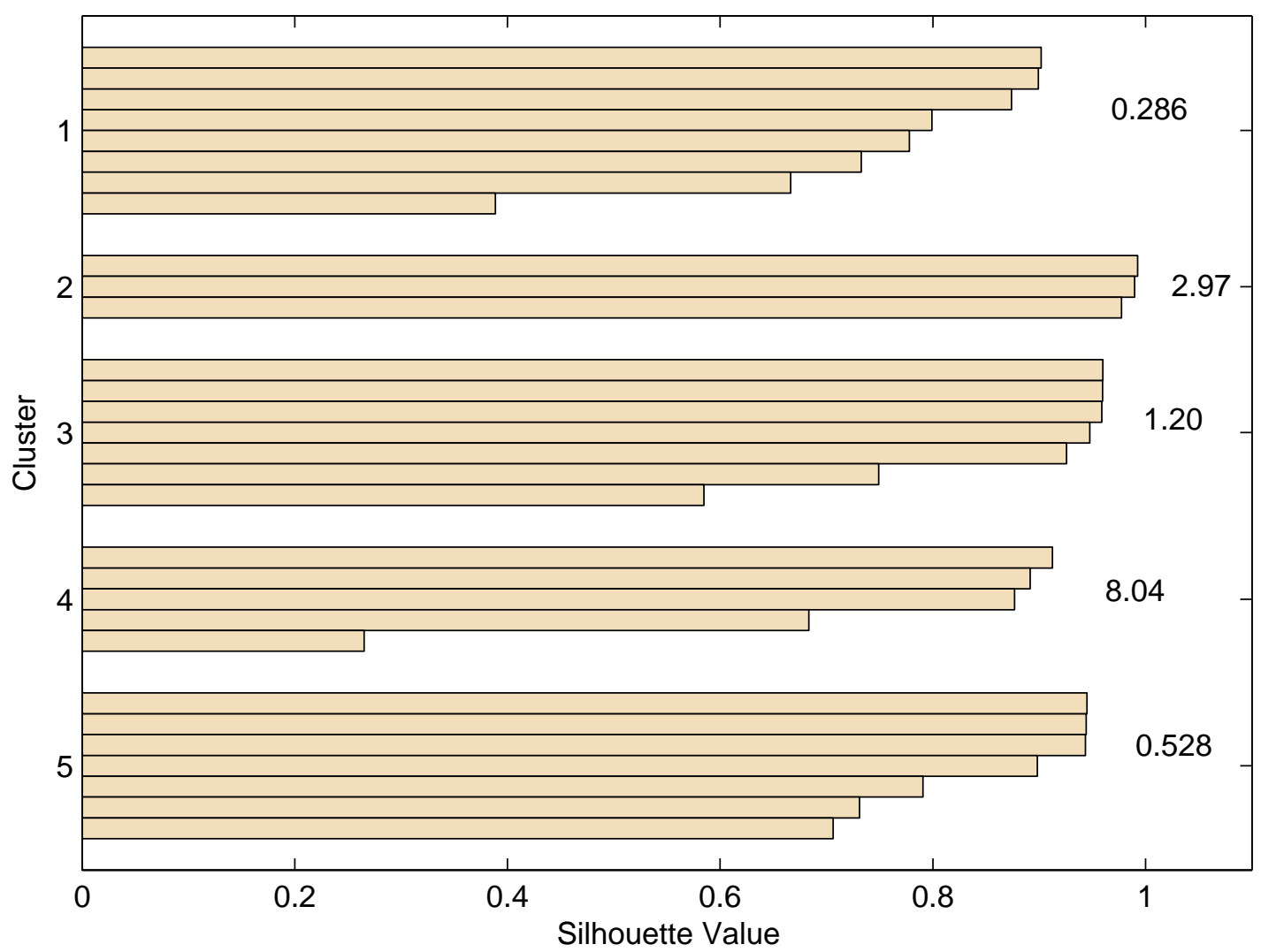

Fig. 3 The silhouette diagram from the K-means clustering algorithm that minimizes Euclidean squared distances. The algorithm finds five clusters of pulsars (see Table 1). The silhouette values are very large $(\geq 0.6)$ for nearly all of the pulsars, indicating that the clusters are well-separated. The centroids of the clusters are also shown, and they are in excellent agreement with the group centers determined from the raw data (see Figure 2).

We also constructed the "silhouette diagram" of the data (Rousseeuw 1987; Kaufman \& Rousseeuw 1990), as shown in Figure 3 . This diagram shows the significance of clustering. The silhouette values of nearly all of the pulsars are very large $(\geq 0.6)$, indicating that the clusters are well-separated with no overlap at all; and only two members (in clusters 1 and 4, respectively) are located at the outskirts of their clusters that are otherwise very dense, just like clusters 2,3 , and 5 . These two outliers have positive silhouette values, thus they cannot be moved to the nearest neighboring cluster.

The two lowest clusters in $\mu$ shown in Figure 2 are separated by a gap of only 0.08 , so one might think that they may be merged into one group. We ran the K-means algorithm seeking only 4 clusters, but in this case the quality of the results was degraded. The silhouette diagram showed 7 outliers of which 4 were in the merged cluster with centroid $0.37 \pm 0.17$.

The dense clustering seen in the above figures is not by itself evidence that the Magellanic pulsars are segregated into five well-defined groups. If one chooses $30 \log \mu$ values in $(-1,1)$ from a uniform random distribution and distributes them into 20 bins, some groups are bound to appear by chance. But 
when the known spin periods are also randomly assigned to these points, the resulting $P_{S}-L_{X}$ diagrams do not resemble the distribution of the observed $L_{X}$ values versus spin period. In particular, the numerical experiments do not reproduce the lowest propeller line found for $\mu=3 \times 10^{29} \mathrm{G} \mathrm{cm}^{3}$ or the theoretical relation (Stella et al. 1986) within each group (see Section 1.2 below).

To rectify this problem, we limited the $L_{X}$ parameter space between the lowest propeller line (Christodoulou et al. 2016) and the canonical Eddington line $\left(L_{E d d}=1.8 \times 10^{38} \mathrm{erg} \mathrm{s}^{-1}\right)$. In that case, the groups at high spin periods were wiped out because of the large ranges in $L_{X}$ values that occur at higher spin periods. Therefore, these random distributions also do not resemble the demarkation shown in Table1, especially in the cases Highest to Third.

\subsection{Outline}

Irrespective of clustering arguments, the above five clusters of pulsars will be physically meaningful only if, in addition, they obey the theoretical relation for the propeller line (Stella et al. 1986). In Section 2 , we describe our investigation of such additional propeller lines and their comparison with theoretical predictions. In Section 3] we summarize and discuss our results.

\section{PROPELLER LINES AND MAGNETIC FIELDS IN THE MAGELLANIC CLOUDS}

We process the data (from XMM-Newton, Chandra, and the published literature) presented in Christodoulou et al. (2016) as follows: First we remove the upper limits in cases of no detection and the few extremely faint (and uncertain) detections that fell below the lowest propeller line which may represent unpulsed magnetospheric emission (Campana et al. 1995; Corbet 1996; Campana 1997), if they are real. Then we follow an iterative process: we remove all the observations of the sources that defined the lowest propeller line and we consider the faintest observations that appear to define the next higher propeller line according to Figures 2 and 3 . In each subsequent step, we remove all the observations of the sources that define lower-lying propeller lines and we consider the next cluster of pulsars. The iteration continues until all the data are exhausted. This process results in the five dense groups of pulsars discussed above (Table 1 and Figure 4).

For each of the five groups, we carry out a linear regression of the faintest observations in the $\log P_{S}$ $\log L_{X, \min }$ diagram in order to determine how close each best-fitted slope is to the theoretical value of $-7 / 3$ (Table 2). The precise theoretical propeller line is obtained from the equation given by Stella et al. (1986) and for the canonical pulsar parameters $M=1.4 M_{\odot}$ and $R=10 \mathrm{~km}$, viz.

$$
L_{X, \min } \approx 2 \times 10^{37}\left(\frac{\mu}{10^{30} \mathrm{G} \mathrm{cm}^{3}}\right)^{2}\left(\frac{P_{S}}{1 \mathrm{~s}}\right)^{-7 / 3} \mathrm{erg} \mathrm{s}^{-1}
$$

This equation contains only one free parameter, the magnetic moment of the pulsar $\left(\mu \equiv B R^{3}\right.$, as was defined by Stella et al. 1986) assuming a dipolar magnetic field of magnitude $B$ on its surface. We note that the magnetic moments determined from the Stella et al. (1986) equation are subject to variations of no more than $54 \%$ if large noncanonical values are used and that most of the uncertainty comes from the pulsar radius: when we adopt $\Delta M=0.6 M_{\odot}$ and $\Delta R=8 \mathrm{~km}$ (Lattimer \& Prakash 2001) for the variation of $\mu \sim M^{1 / 3} R^{1 / 2}$, we find that $\Delta \mu / \mu=54 \%$ in which only $14 \%$ is contributed by the variation of the mass.

Table 2 shows that the four lower best-fitted lines are in excellent agreement with the corresponding theoretical propeller lines (the null hypothesis is rejected at the 95\% confidence level). Only for the fifth and highest line, for which we used the last five remaining objects, is the error in the slope substantial (about 20\% 1], although its $p$-value is still significant. To make up for this deviation in slope, we produced another linear fit to the data in which we fixed the intercepts to the values that yield a slope of $-7 / 3$

\footnotetext{
${ }^{1}$ Even in this case, when we include the published observations of pulsars with spin periods $P_{S}>100 \mathrm{~s}$, we find only two more propeller states (SXP131, $L_{X}=2.7 \times 10^{34} \mathrm{erg} \mathrm{s}^{-1}$; SXP342, $L_{X}=3.8 \times 10^{33} \mathrm{erg} \mathrm{s}^{-1}$; Lavcock et al. 2010), and the slope $(-2.358 \pm 0.179)$ returns gracefully to a value that differs from $-7 / 3$ by only $1 \%$; then the intercept $(39.244 \pm 0.290)$ gives a magnetic field of $B=9.4 \mathrm{TG}\left(r^{2}=0.972, p=0.048\right)$ for the highest propeller line.
} 


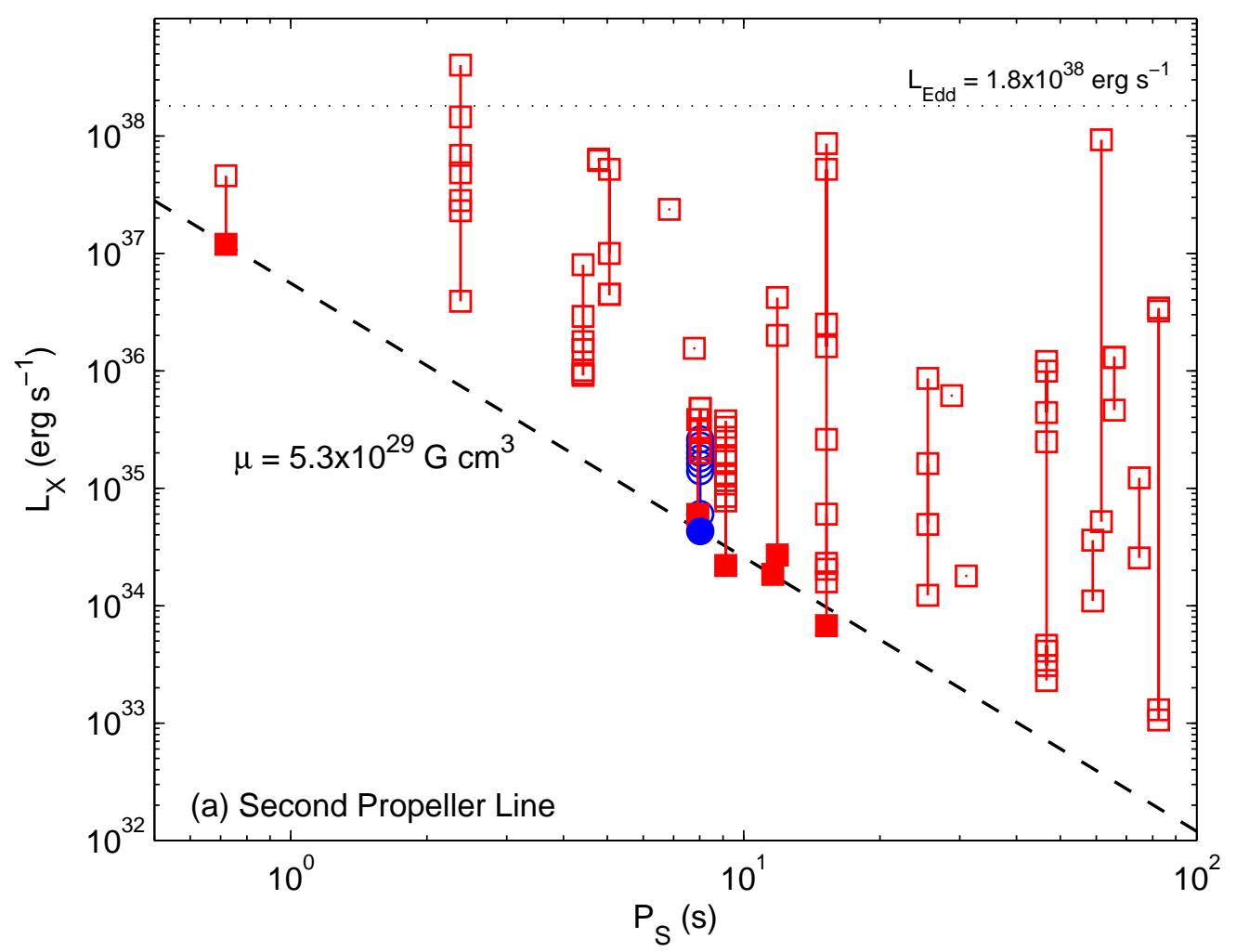

Fig. $4 L_{X}$ vs. $P_{S}$ diagram for the X-ray sources listed in Table 1 The data were presented in Christodoulou et al. (2016) and the observations of the sources that defined the lowest propeller line have been removed. In each iteration from (a) to (d), the observations of the pulsars defining lower-lying propeller lines are removed. Unfilled symbols represent individual observations. Dots mark multiple observations with nearly identical results. Filled symbols in each panel represent the pulsars that define the corresponding propeller line listed in Tables 1 and 2. Blue circles in panel (a) represent observations of the single pulsar CXOU J010043.1721134. The dashed line is the theoretical propeller line with a slope of $-7 / 3$ that implies the magnetic-moment value shown in each panel. The dotted line is the Eddington luminosity for a $1.4 M_{\odot}$ pulsar.

exactly. These results are shown in Table 3, The correlation coefficients $\left(r^{2}\right)$ indicate that these fits are also of high quality. The $p$-values of these fits are extremely small, but they are meaningless because of the imposed constraint. However, even the highest propeller line with a constrained slope of $-7 / 3$ is an acceptable fit to the few remaining data points $\left(r^{2}=0.966\right)$.

The magnetic moments listed in Table 3 were then determined from the intercepts of the fits using equation (1). The corresponding magnetic-field magnitudes were also determined using the definition $\mu \equiv B R^{3}$ and the canonical pulsar radius of $R=10 \mathrm{~km}$. The range of magnetic fields $(B \approx 0.3-7.3 \mathrm{TG})$ is consistent with the values quoted in the literature for many Galactic and extragalactic HMXBs; those obtained by applying accretion theory (Stella et al. 1986, 1994; Campana 1997; Corbet et al. 1997; Galache et al. 2008; Bachetti et al. 2014); and those obtained by NuSTAR observations of cyclotron resonance features in the 14-32 keV energy range (Tendulkar et al. 2014; Brightman et al. 2016). 


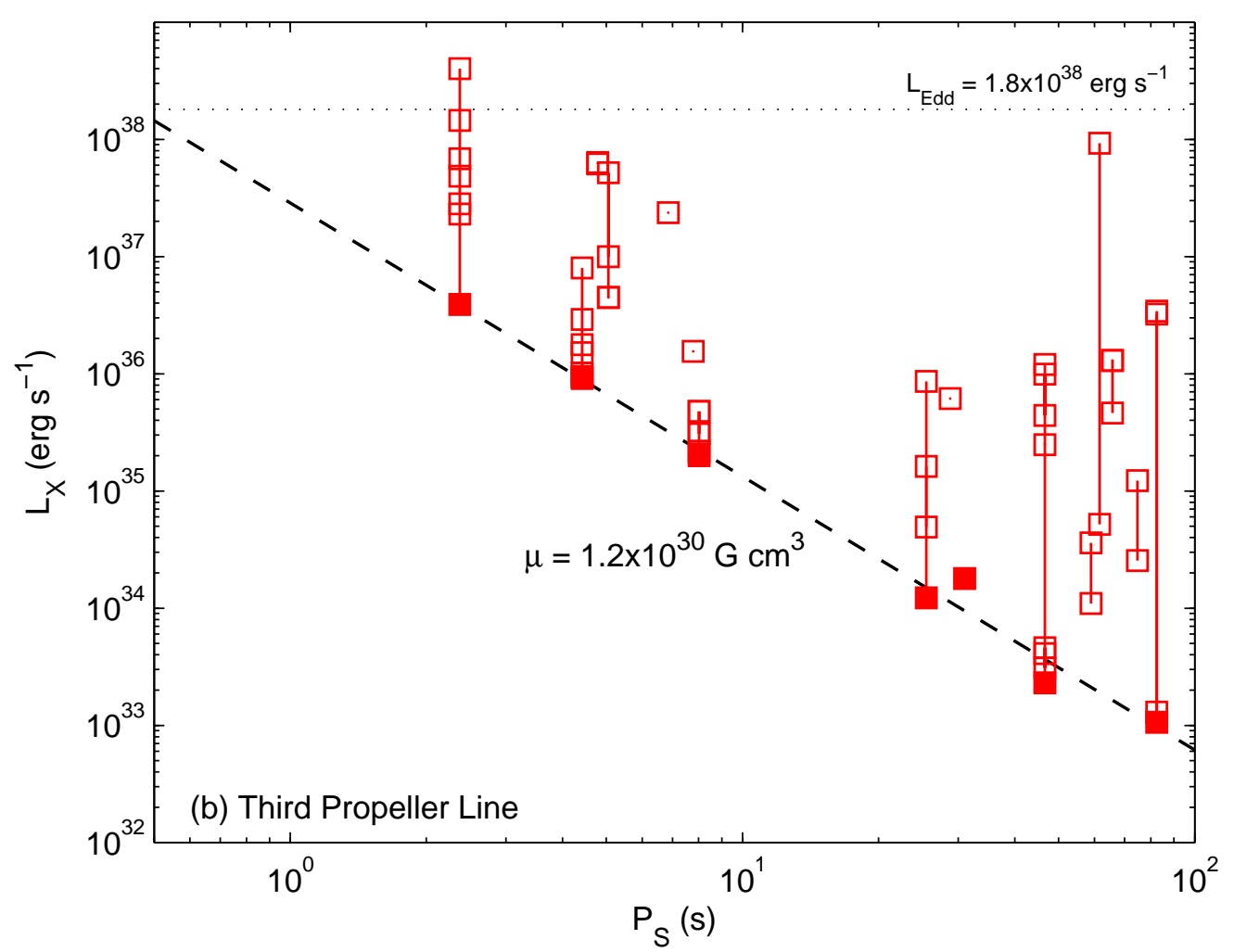

Table 2 Linear Regressions for the 5 Propeller Lines

\begin{tabular}{ccccccc}
\hline $\begin{array}{c}\text { Propeller } \\
\text { Line }\end{array}$ & $\begin{array}{c}\text { Number } \\
\text { of MC Pulsars }\end{array}$ & $\begin{array}{c}\text { Slope } \\
( \pm 1 \sigma \text { Error })\end{array}$ & $\begin{array}{c}\text { Deviation } \\
\text { from }-7 / 3 \text { Slope }\end{array}$ & $\begin{array}{c}\text { Intercept } \\
( \pm 1 \sigma \text { Error })\end{array}$ & $\begin{array}{c}p \text {-value } \\
\text { Correlation } \\
\left(r^{2}\right)\end{array}$ \\
\hline Highest & 5 & $-2.795( \pm 0.089)$ & $-19.80 \%$ & $39.725( \pm 0.115)$ & 0.0203 & 0.997 \\
Fourth & 3 & $-2.445( \pm 0.001)$ & $-4.80 \%$ & $38.371( \pm 0.001)$ & 0.0002 & 1.000 \\
Third & 7 & $-2.334( \pm 0.115)$ & $-0.04 \%$ & $37.458( \pm 0.142)$ & 0.0313 & 0.988 \\
Second & 7 & $-2.347( \pm 0.131)$ & $-0.60 \%$ & $36.758( \pm 0.116)$ & 0.0355 & 0.985 \\
Lowest & 8 & $-2.322( \pm 0.092)$ & $+0.47 \%$ & $36.207( \pm 0.088)$ & 0.0252 & 0.991 \\
\hline
\end{tabular}

Table 3 Linear Fits Imposing a Slope of $-7 / 3$ to the Data

\begin{tabular}{ccccc}
\hline \hline $\begin{array}{c}\text { Propeller } \\
\text { Line }\end{array}$ & $\begin{array}{c}\text { Intercept } \\
\text { (for slope } \equiv-7 / 3)\end{array}$ & $\begin{array}{c}\text { Correlation } \\
\left(r^{2}\right)\end{array}$ & $\begin{array}{c}\mu \\
\left(\mathrm{G} \mathrm{cm}^{3}\right)\end{array}$ & $\begin{array}{c}B \\
(\mathrm{TG})\end{array}$ \\
\hline Highest & 39.029 & 0.966 & $7.3 \times 10^{30}$ & 7.3 \\
Fourth & 38.224 & 0.998 & $2.9 \times 10^{30}$ & 2.9 \\
Third & 37.456 & 0.988 & $1.2 \times 10^{30}$ & 1.2 \\
Second & 36.744 & 0.985 & $5.3 \times 10^{29}$ & 0.53 \\
Lowest & 36.224 & 0.991 & $2.9 \times 10^{29}$ & 0.29 \\
\hline
\end{tabular}

\section{SUMMARY AND DISCUSSION}

We have processed the Magellanic HMXB data from the XMM-Newton and the Chandra archives and from the published literature that were listed in Christodoulou et al. (2016) who determined the lowest 


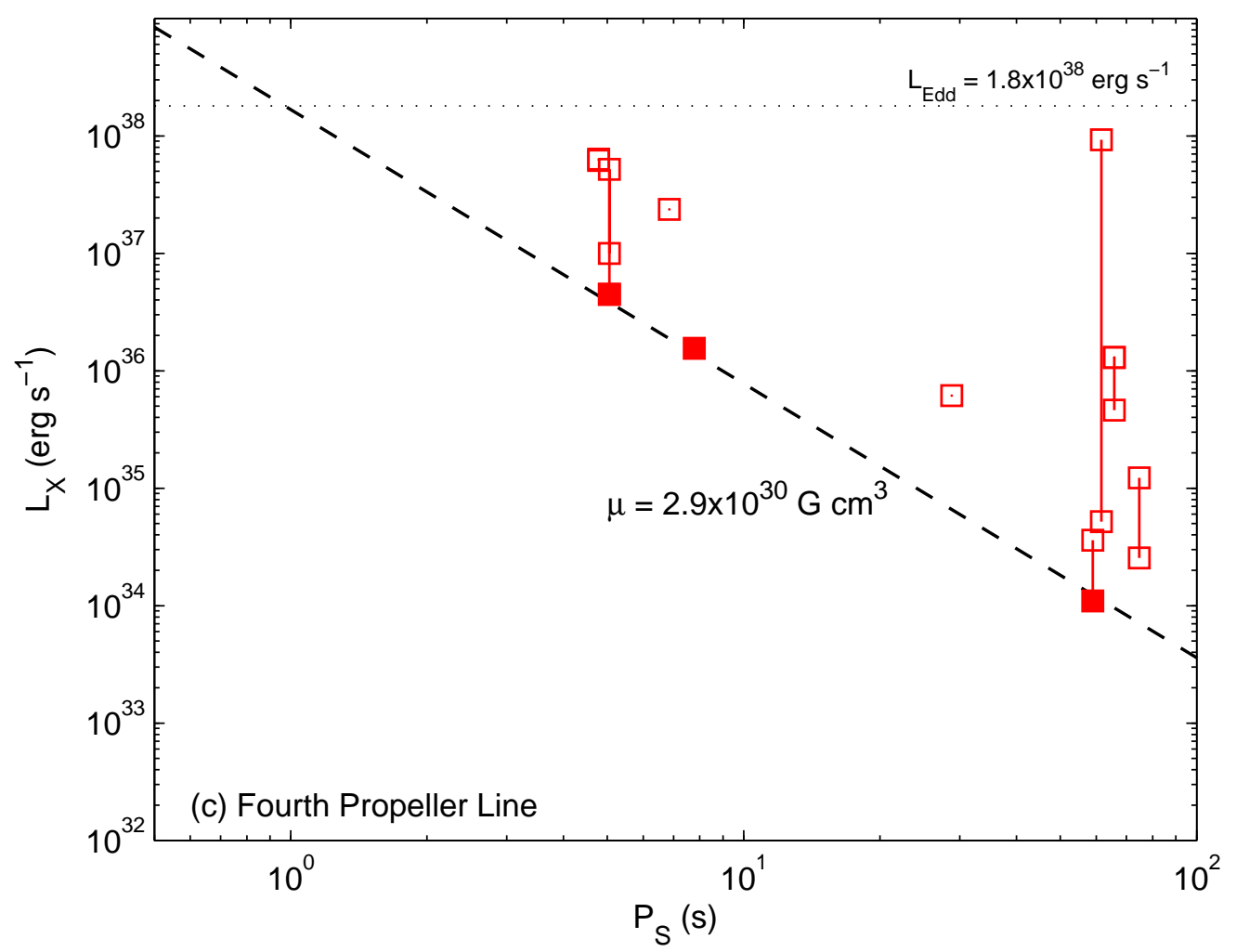

propeller line in the spin period-luminosity $\left(P_{S}-L_{X}\right)$ diagram on which accretion proceeds at the smallest possible rates and the X-ray emission is pulsed. We used the K-means clustering algorithm (Seber 1984; Spath 1985) followed by an iterative process in order to search for additional propeller lines and we found a total of five such lines for pulsars with $P_{S}<100 \mathrm{~s}$ (Tables 1 and 2 , Figures 2 and 4 ). The linear regressions of four data sets produced best-fitted lines of high quality that are additionally in excellent agreement with the theoretical $P_{S}-L_{X}$ relation of Stella et al. (1986). The fifth data set that resulted in the highest propeller line can also be fitted with the theoretical relation to a satisfactory degree (Table 3).

The linear fits of the theoretical relation (equation [1]) were used to determine the magnetic fields of the five groups of pulsars. The entire population of MC pulsars is described by the propeller lines shown in Table 3 and the corresponding magnetic-field magnitudes $(0.29,0.53,1.2,2.9$, and 7.3 , in units of TG). These discrete values may come as a surprise because there is no a priori reason for the lowluminosity X-ray observations of all of these objects to line up on precise straight lines such as those shown in Tables 2 and 3 and in Figure 4, unless of course several different factors concur:

(a) the X-ray duty cycles of the pulsars were very well monitored during all of these 15 years;

(b) the observations did manage to probe the lowest levels of pulsed X-ray emission from each pulsar; and

(c) the physical characteristics of the X-ray emitting regions, the accretion processes, and the pulsars themselves are very similar for the entire population (as was also found by Coe et al. 2010).

It will be interesting to see if these five groups of MC pulsars (Table 1) do indeed provide robust classes based on their surface magnetic fields, as more of their physical properties will be obtained in the following years that will produce more classifications of these objects. We believe that our classification of Magellanic pulsars will prove very useful to such future investigations. 


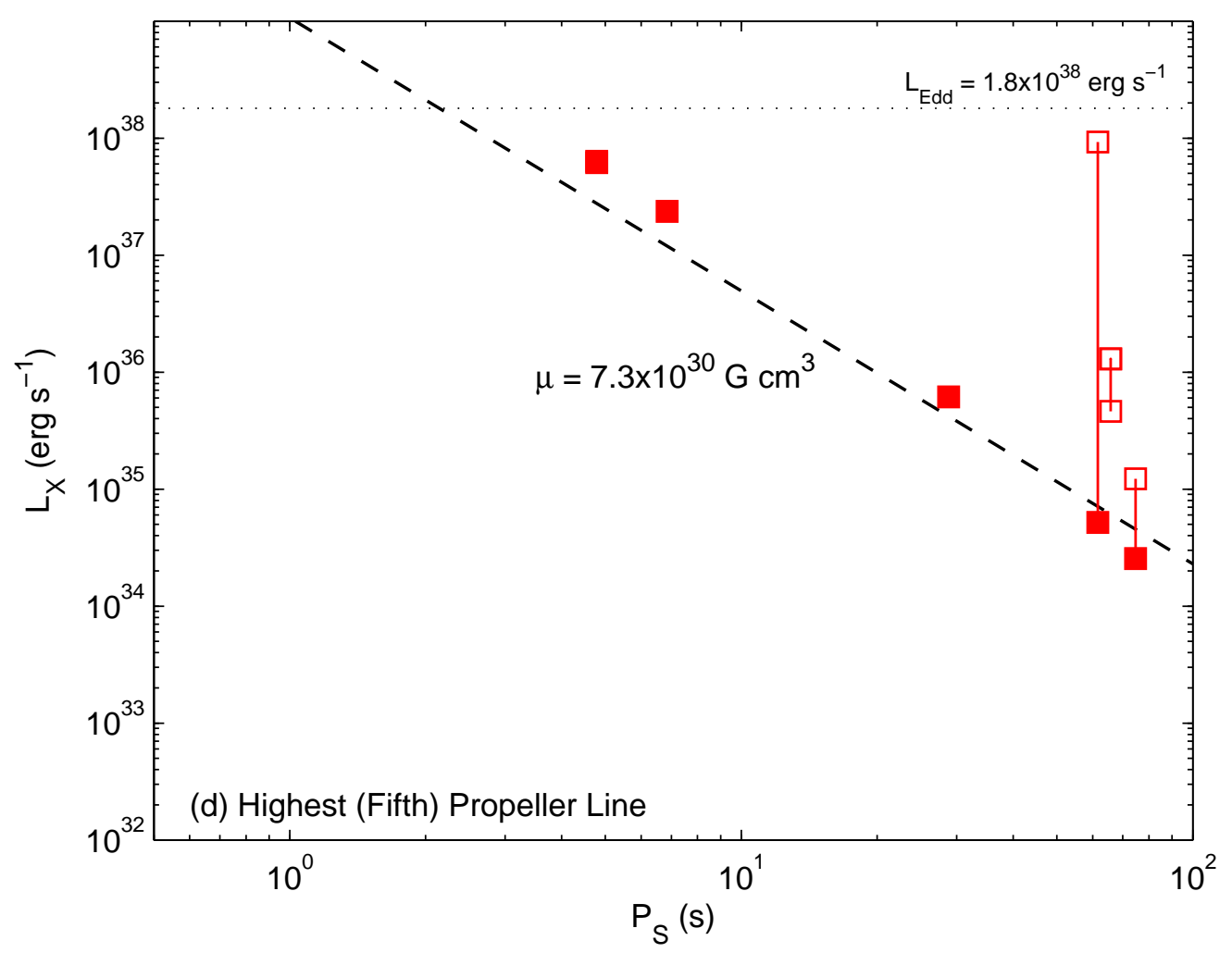

Acknowledgements This work was supported by NASA grant NNX14-AF77G.

\section{References}

Bachetti, M., Harrison, F. A., Waltron, D. J., et al. 2014, Nature, 514, 202

Brightman, M., Harrison, F., Walton, D. J., et al. 2016, ApJ, 816, 60

Campana, S. 1997, A\&A, 320, 840

Campana, S., Stella, L., Mereghetti, S., \& Colpi, M. 1995, A\&A, 297, 385

Christodoulou, D. M., Laycock, S. G. T., Yang, J., \& Fingerman, S. 2016, ApJ, 829, 30

Coe, M. J., McBride, V. A., \& Corbet, R. H. D. 2010, MNRAS, 401, 252

Corbet, R. H. D. 1996, ApJ, 457, L31

Corbet, R. H. D., Charles, P. A., Southwell, K. A., \& Smale, A. P. 1997, ApJ, 476, 833

Galache, J. L., Corbet, R. H. D., Coe, M. J., Laycock, S., \& Schurch, M. P. E. 2008, ApJS, 177, 189

Kaufman L., \& Rousseeuw, P. J. 1990, Finding Groups in Data: An Introduction to Cluster Analysis, Hoboken, NJ: John Wiley \& Sons

Lattimer, J. M., \& Prakash, M. 2001, ApJ, 550, 426

Laycock, S., Zezas, A., Hong, J., Drake, J. J., \& Antoniou, V. 2010, ApJ, 716, 1217

Rousseeuw, P. J. 1990, J. Comp. Appl. Math., 20, 53

Seber, G. A. F. 1984, Multivariate Observations, Hoboken, NJ: John Wiley \& Sons

Spath, H. 1985, Cluster Dissection and Analysis: Theory, FORTRAN Programs, Examples (Translated by J. Goldschmidt), New York: Halsted Press

Stella, L., Campana, S., Colpi, M., et al. 1994, ApJ, 423, L47

Stella, L., White, N. E., \& Rosner, R. 1986, ApJ, 308, 669

Tendulkar, S. P., Fürst, F., Pottschmidt, K., et al. 2014, ApJ, 795, 154 
Yang, J., Laycock, S. G. T., Christodoulou, D. M., et al. 2017, ApJ, in press 